\title{
The rapid generation of chimerical genes expanding protein diversity in zebrafish
}

\author{
Beide Fu' ${ }^{1,2}$, Ming Chen ${ }^{1,2}$, Ming Zou ${ }^{1,2}$, Manyuan Long ${ }^{3}$, Shunping He ${ }^{1 *}$
}

\begin{abstract}
Background: Variation of gene number among species indicates that there is a general process of new gene origination. One of the major mechanism providing raw materials for the origin of new genes is gene duplication. Retroposition, as a special type of gene duplication- the RNA-based duplication, has been found to play an important role in new gene evolution in mammals and plants, but little is known about the process in the teleostei genome.

Results: Here we screened the zebrafish genome for identification of retrocopies and new chimerical retrogenes and investigated their origination and evolution. We identified 652 retrocopies, of which 440 are intact retrogenes and 212 are pseudogenes. Retrocopies have long been considered evolutionary dead ends without functional significance due to the presumption that retrocopies lack the regulatory element needed for expression. However, 437 transcribed retrocopies were identified from all of the retrocopies. This discovery combined with the substitution analysis suggested that the majority of all retrocopies are subject to negative selection, indicating that most of the retrocopies may be functional retrogenes. Moreover, we found that 95 chimerical retrogenes had recruited new sequences from neighboring genomic regions that formed de novo splice sites, thus generating new intron-containing chimeric genes. Based on our analysis of 38 pairs of orthologs between Cyprinus carpio and Danio rerio, we found that the synonymous substitution rate of zebrafish genes is $4.13 \times 10^{-9}$ substitution per silent site per year. We also found 10 chimerical retrogenes that were created in the last 10 million years, which is 7.14 times the rate of 0.14 chimerical retrogenes per million years in the primate lineage toward human and 6.25 times the rate of 0.16 chimerical genes per million years in Drosophila. This is among the most rapid rates of generation of chimerical genes, just next to the rice.
\end{abstract}

Conclusion: There is compelling evidence that much of the extensive transcriptional activity of retrogenes does not represent transcriptional "noise" but indicates the functionality of these retrogenes. Our results indicate that retroposition created a large amount of new genes in the zebrafish genome, which has contributed significantly to the evolution of the fish genome.

\section{Background}

Retroposition entails a process in which RNA (including mRNA transcribed from a parent gene) is subsequently reverse-transcribed into cDNA and inserted into a new locus on the chromosome to form a new retrocopy locus. The main characteristic of the retrocopy is the lack of introns, and if the retroposition event is recent enough, a poly(A) tail and short flanking duplicate

\footnotetext{
* Correspondence: heshunping@gmail.com

${ }^{1}$ The Key Laboratory of Aquatic Biodiversity and Conservation of Chinese Academy of Sciences, Institute of Hydrobiology, Chinese Academy of Sciences, Wuhan 430072, PR China

Full list of author information is available at the end of the article
}

sequences can be observed as well[1]. Retrocopies often become processed pseudogenes (retropseudogenes) and are eventually deleted because they do not have the regulatory elements necessary for expression. However, the extensive structural changes in retrocopies have been speculated as "evolutionary seeds" for the evolution of new gene functions if they acquire new regulatory sequences [2] (hereafter called retrogenes). For example, the finding of the jingwei gene in Drosophila yakuba and the sphinx gene in Drosophila melanogaster revealed that the retrogene can recruit a certain regulatory sequence and evolve a new function defined by new expression and new gene structure [3-5]. In the
C Biomed Central

() 2010 Fu et al; licensee BioMed Central Ltd. This is an Open Access article distributed under the terms of the Creative Commons Attribution License (http://creativecommons.org/licenses/by/2.0), which permits unrestricted use, distribution, and reproduction in any medium, provided the original work is properly cited. 
human genome, chimeric retrogenes may originate from small nuclear RNAs and mRNAs by RNA-RNA recombination [6-10]. The structures of such functional retrogenes are usually chimerical: they may either recruit a new regulatory element from the insertion site to form a peculiar coding region that evolved from difference sources $[5,11]$, or they can use exons from genes near the insertion site to form a new gene structure [12,13]. Such chimerical structures usually confer a new function or a new expression pattern that parental genes do not have, thus often leading to adaptive evolution [2,14]. For example, retrogenes in the Drosophila genome show a pattern that escaped from the $\times$ chromosome to the autochromosome to evolve new testis functions $[11,15]$. The e(y) 2 retrogene in Drosophila melanogaster performs a general function and is ubiquitously expressed, while the source gene $e(y) 2 b$ is functional only in a small group of male germ cells [16]. These studies support the hypothesis that retrogenes played an important role in the evolution of functionality in organisms.

Large numbers of retrogenes have been found in mammal, plant and insect genomes, but few retrogenes have been identified and studied in fish genomes $[11,15,17-20]$. The first retrogene that was reported in the fish genome was an ocular rod opsin gene, rho, which does not have introns, unlike the other introncontaining rod opsin genes in vertebrates, errlo, [21]. Previous studies revealed that in the mammal genome, the enzymatic machinery of LINE1 (Long Interspersed Nuclear Element 1, L1) is responsible for the creation of retrogenes [22]. L1 s are widely present in mammals and account for up to about $25 \%$ of the genome $[23,24]$. With the sequenced zebrafish genome, many lineages of L1 s were identified [24,25], therefore we predict that there may be a large amount of retrogenes in the zebrafish genome. Recently, a significant number of transcribed and functional retrocopies were discovered in the primate and rodent genome [11,15,26-29], but there has not been any study of the expressed retrocopies in the teleostei genome.

To understand the functional and evolutionary impact of retroposition in the zebrafish genome, we screened the full genome of zebrafish for retrocopies and chimerical retrogenes. We found that there are 652 retrocopies and 95 chimerical retrogenes in zebrafish genome. This is the first study conducted for retrocopies and chimerical retrogenes in teleostei genome at the whole-genome level.

\section{Results}

\section{Abundant retrogenes in the zebrafish genome}

The 31743 protein sequences downloaded from the Ensembl database were used to detect retrogenes in the zebrafish genome with a computational pipeline with stringent parameters depicted in the flow chart shown in Figure 1. All 31743 protein sequences were mapped on the genome with TblastN [30], and 12504 longest regions mapped with the proteins were retained. Nearby homology matches (distance $<40 \mathrm{bp}$ ), which were not likely separated by introns, were merged after a series of Perl scripts' parsing. The structure of the 12504 sequences was obtained by GeneWise[31], and only 12333 sequences with intron larger than 40 bp were retained. To find the parental gene, the 12333 sequences were mapped with the whole 31743 protein sequences with FASTY[32], and we only kept the 8543 results that had the best score but no overlap with the protein position on the genome. Meanwhile the two sequences had more than $50 \%$ of the gene sequence's length and identified more than $50 \%$ on the amino acid level.

We only included the gene pairs of the Ensembl peptides with multiple coding exon (parental gene) as the best hit of the merged sequence (new gene), as these can prevent the single exon gene, e.g., olfactory receptor genes, from becoming the "parent" gene. Finally, if the sequence of the new gene overlapped two exons of the parental gene and the distance between the two exons was larger than $70 \mathrm{bp}$, we confirmed the new gene as a retrogene. We chose $70 \mathrm{bp}$ as a threshold based on the following: 1) the majority of introns in zebrafish genome are larger than $70 \mathrm{bp}$ [33], so it can exclude small gaps in parental genes annotated as introns by mistake; and 2) we ensured that at least one intron was lost in the new retrogene since $70 \mathrm{bp}$ is larger than the gap size (40 bp) we used in the merging step. Ultimately, 6,021 retrogene candidates were found that satisfied all our criteria. To divide these candidates into primary retrogenes and duplicates that descend from primary retrogenes, we used the GeneWise [31] to determine whether the lost intron is derived from the parental gene. This produced 652 primary retrogenes, out of which 212 retrocopies with either frame-shift mutations or premature stop codons were defined as retropseudogenes. The rest of the retrogenes (440) were defined as intact retrogenes. (See Additional file 1). Actually, the number of primary retrogenes is most likely higher than this sample set, because some intronless copy could have originated through retroposition of old retrogenes.

To obtain the age distribution of all the retrocopy formation events, we plotted the Ks distribution of the parental-retrocopy pairs (Figure 2). Based on the divergence time of Danio rerio and Cyprinus carpio of 50 Mya (million years ago) [34], we used 38 pairs of orthologs between the two fishes, and found that the synonymous substitution rate of zebrafish genes is $4.13 \times 10^{-9}$ substitution per silent site per year. We found that the majority of retrocopies formed within the past 50 million years, indicating that recent, rapid 


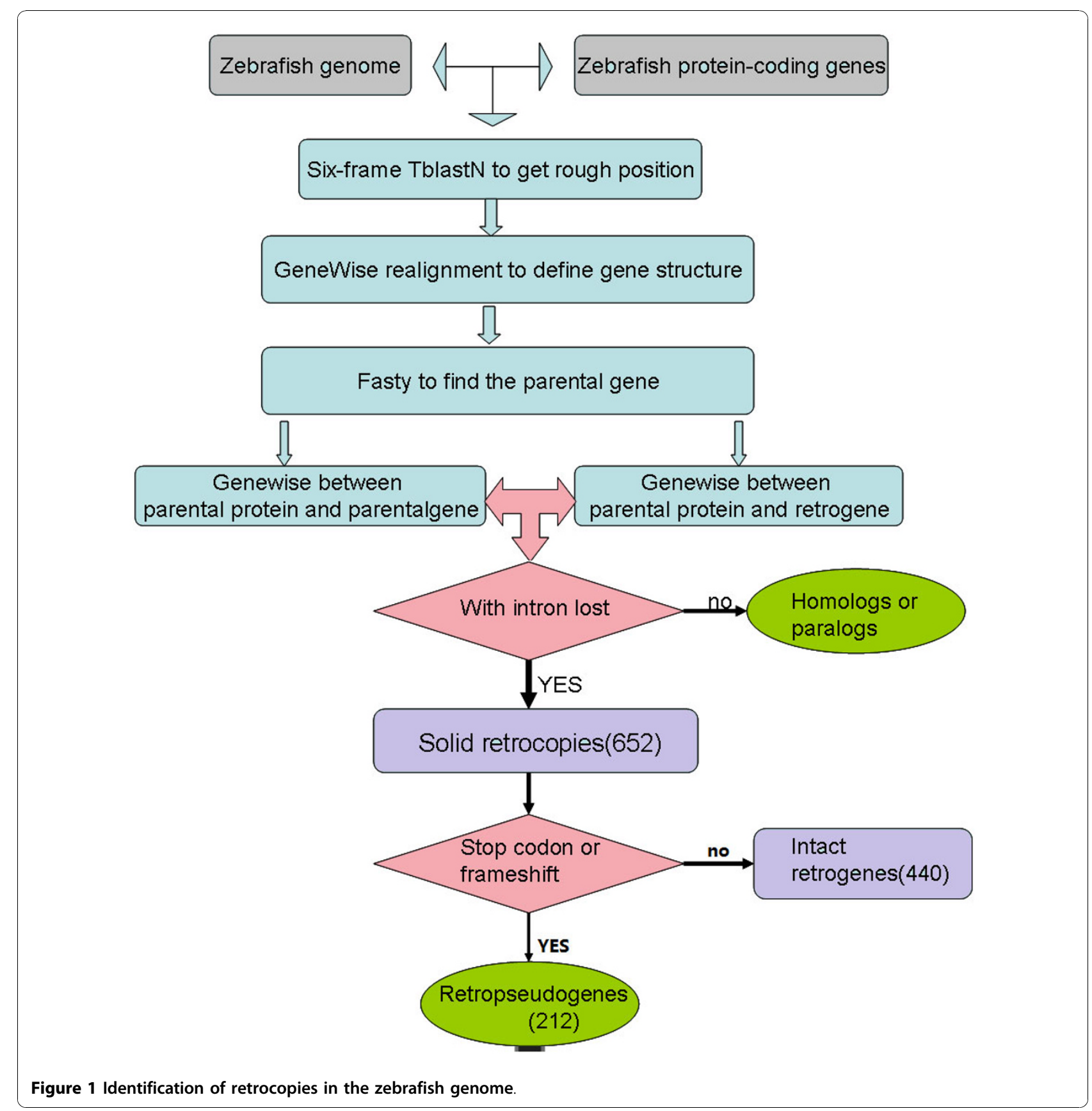

formation of retrocopies may not have only occurred in primate lineages $[29,35]$.

\section{Large amount of transcribed retroposed genes in the zebrafish genome}

Generally, retrocopies were thought of as sequences without transcriptional ability, but many transcribed retrogenes were found in the primate and rodent genomes [11,15,26-29]. To explore whether this pattern exists in the zebrafish genome, we used ESTs to represent the transcription ability of these retrogenes, because they provide better discrimination within close paralogs than short-tag expression sequences or data from hybridization-based methods $[36,37]$. To map ESTs to the retrogenes, we used the rigorous pipeline of Vinckenbosch et al. (2006) that excludes erroneous mapping to parental genes or other paralogs (see methods). These analyses showed that about two-thirds of retrocopies (437 of 652 or $67 \%$ ) matched with at least one EST, revealing that the majority of retrogenes are transcribed. (See Additional file 2) This proportion is much higher than that in human genome (1080 of 3590, or $30.1 \%$ ). This might 


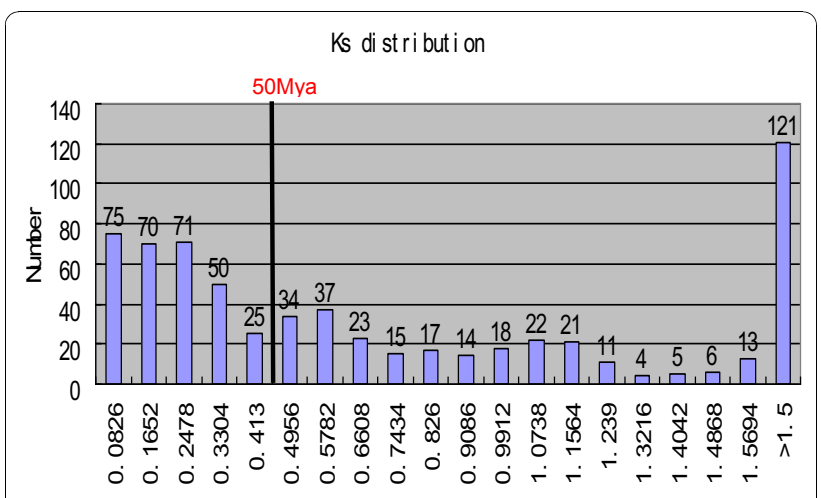

Ks

Figure $2 \mathrm{Ka} / \mathrm{Ks}$ ratios in retrogene between the retrogenes and its parental sequences

be because the retropseudogenes were about $84 \%$ of all the retrogenes (3015 of 3590 , or $83.9 \%$ ), but the expression of the retropseudogenes in human genome $(26.8 \%)$ is much lower than in zebrafish genome $(60 \%$, see below)[38].

We investigated how the new retrogenes recruited new regulatory sequences. We fully screened the genomic context of retrogenes to explore the potential donors of the regulatory elements. We first compared the number of transcribed retrogenes located inside and outside of other genes. In the 440 intact retrogenes, we found that 198 were inserted into "host" genes and 242 between the two previous existing genes. Among the 198 retrogenes within a gene, 152 (76.7\%) had at least one EST, suggesting they recruited the regulatory sequences from parental genes. Interestingly, this proportion of $76.7 \%$ was higher than the proportion of transcribed intergenic retrogenes (285 of 454 , or $62.7 \%$ ), indicating the retrogenes inserted inside a gene were more likely transcribed as a consequence of recruitment of a cis-regulatory element from the host gene.

For the retrogenes that were located outside a gene, we hypothesized that these retrogenes were transcribed because they were inserted into a genomic region that contained a transcriptionally more active region with more potentially active regulatory sequences. Such a retrosequence would be more easily to pick up regulatory elements. By contrast, if a retrosequence were inserted into a transcriptionally inactive genomic region with less potentially active regulatory sequences, then the retrogenes would be less likely to pick up a regulatory sequence and become processed pseudogenes. A prediction based on this hypothesis is that the regions flanking functional retrogenes would transcribe more RNAs; the regions flanking processed pseudogenes would transcribe less RNAs. We tested whether regions surrounding transcribed retrogenes are more active than regions surrounding transcribed silent retrogenes. We compared the number of ESTs mapped to the surrounding regions, in which transcribed retrogenes have more ESTs (median number of ESTs, 76) than transcribed silent retrogenes (median number of ESTs, 28), and found that the difference was statistically significant. $\left(\mathrm{P}<10^{-5}\right.$, MannWhitney U test).

\section{Most zebrafish retrogenes are very likely to be functional new genes}

In the 652 primary zebrafish retrogenes, we only found 212 processed pseudogenes (32\%), which contained frame shift mutations or premature stop codons. We further tested the functionality of the rest of the 440 (68\%) retrogenes by using two approaches.

First, it is likely that a pseudogene without function may sometimes also be transcribed [39]. Hence, the previous test of transcription of intact retrogenes ought to be further examined to test their functionality. We compared the transcription patterns between the 440 retrogenes with intact ORF and 212 retropseudogenes. We found that the percentage of intact retrogenes with at least one EST (308 of 440 or $70 \%$ ) was significantly higher than that of pseudogenes (129 of 212 or 60\%) $(\mathrm{P}<0.05$, Fisher's exact test). The more significant pattern was observed (Table 1) when we used mRNA as transcription evidence (127 of 440 intact $v s 10$ of 212 pseudogene, $\mathrm{P}<10^{-14}$, Fisher's exact test). These data show that intact retropseudogenes are likely expressed and functional, compared to the hypothetical processed pseudogenes. It also should be noted here that the two criteria of disabled mutations, frameshift or nonsense mutations, to annotate a processed pseudogene may be too stringent, because it has been found that some functional genes can be formed by splicing out the gene regions that harbor frameshift and nonsense mutation as new introns $[40,41]$.

An overview of the highly transcribed retrogenes (as judged by the number of matching ESTs) also offers compelling evidence for transcription as a marker of retrogene functionality (Additional file 2). Among the 50 most highly transcribed retrogenes, the vast majority (39 of 50 or $78 \%$ ) was intact, whereas only a small part (11

$\begin{aligned} & \text { Table } 1 \text { Retrogenes transcription supported by } \\
& \text { expression data }\end{aligned}$
\begin{tabular}{cll}
\hline $\begin{array}{c}\text { Expressed } \\
\text { info }\end{array}$ & $\begin{array}{l}\text { Retrogene type } \\
\text { (number) }\end{array}$ & $\begin{array}{l}\text { Number(percentage in this } \\
\text { type) }\end{array}$ \\
\hline mRNA & Intact(440) & $127(28.86 \%)$ \\
& Pseudo(212) & $10(4.72 \%)$ \\
EST & Intact(440) & $308(70.00 \%)$ \\
& Pseudo(212) & $129(60.85 \%)$ \\
\hline
\end{tabular}


of 50 or $22 \%$ ) was pseudogenes. A similar result was obtained in an extended analysis with the 100 most highly transcribed retrogenes in which 77 of 100 were intact retrogenes. Similarly, many retrogenes with a number of ESTs have been previously identified as functional genes.

Second, we investigated the sequence constraint created by functionality through a comparison of nonsynonymous(Ka) and synonymous(Ks) substitution rates $(\mathrm{Ka} / \mathrm{Ks}$ ratios) between retrogenes and their parental genes. Generally, the evolution of pseudogenes is under neutrality, which means that the $\mathrm{Ka} / \mathrm{Ks}$ values are larger than the ratio of genes subject to functional constraint under purifying selection, and smaller than the ratio of genes under positive selection [42]. This method has been widely used in identifying the functionality of genes $[1,43,44]$. We found that processed pseudogenes have much higher $\mathrm{Ka} / \mathrm{Ks}$ values than intact retrogenes, illustrating that intact retrogenes are subjected to functional constraint. In the comparison between retrogenes with unknown constraints and parental genes that are known to be functional, a stricter criterion should be $\mathrm{Ka} / \mathrm{Ks}<0.5$ to identify functionality of the retrogenes $[11,15]$. We found that a $\mathrm{Ka} / \mathrm{Ks}$ ratio of $65 \%$ (284 cases) of intact retrogenes was significantly lower than 0.5 . These data suggested that the intact retrogenes are under stronger purifying selection and are likely functional. The data also suggested that the processed pseudogenes are also subject to sequence constraint, showing that some of them are either functional in splicing out the disabled mutations or have been functional but recently became pseudogenes by disabled mutations.

\section{High proportion of chimerical genes}

In contrast to rare recent chimerical retrogenes in mammalian genomes $[12,45,46]$, zebrafish retrogenes recruit not only novel regulatory elements but also new coding sequences from the insertion sites and thus give birth to many chimerical genes that translate hybrid proteins. Out of the 440 intact zebrafish retrogenes, 95 (22\%) were predicted to have chimerical protein coding sequence (CDS) structures (See Additional file 3). Among these cases, 26 retrogenes were added with a new peptide to the $C$ terminus, another 26 retrogenes were added with a new peptide to the $\mathrm{N}$ terminus, whereas in 32 other retrogenes, both of the $\mathrm{N}$ and $\mathrm{C}$ terminus were added with a new peptide. For example, in the retrogene ENSDARP00000042310_10_38722825_38723706, both of the two termini were added with a new peptide, while in ENSDARP00000054044_18_29188505_29189548, a new peptide was added to its $\mathrm{N}$ terminus and in ENSDARP00000062080_24_39945989_39946729, a new peptide was added to its $C$ terminus. Furthermore, in the retrogene ENSDARP00000059483_15_3816795_3817442, a part of the $\mathrm{N}$ terminus was changed to 5'UTR in the new hybrid protein structure (Figure 3 ).

All of the 84 retrogenes above were treated as coding fusion with neighboring genes. Whereas in the rest of the 11 retrogenes, 8 recruited $5^{\prime}$ and 3'UTR to form a new single exon gene and 3 retrogenes cover the full length of the new chimerical gene, which were thought to be de novo chimerical genes. This pattern is much different from the pattern within the human genome, which has 36 fusion and 27 de novo chimerical retrogenes[38] ( $\mathrm{P}<0.01$, chi-square test).

In the 95 hybrid new genes, 30 retrogenes overlap with the exon-intron boundary, which indicated that new splice sites were created during the evolutionary history of the retrogenes. A possible reason was that during evolution, new introns were produced in the original transcripts that included the retrogenes[47,48] or new splice sites were created from previous exon regions of the parental genes[41].

There was considerable expression evidence that some of the zebrafish hybrid genes might have acquired new protein functions. To further study the transcription of these hybrid genes, we used full-length mRNA and EST to blast against the transcript of the hybrid genes, and found that 73 hybrid genes have transcription evidence. Combined with the EST data, 26 chimerical retrogenes were among the top 100 transcribed retrogenes. The evidence above illustrated that most of the chimerical retrogenes were functional. Because of the extensive structural variation, these new chimerical genes were under the evolutionary process of neofunctionalization.

To explore the rate of creation of chimerical retrogenes, we counted the Ks distribution of the chimerical retrogenes that were under 1.5. Among the 62 retrogenes, ten of them had Ks values lower than 0.0826 , which means 10 chimerical retrogenes formed in the last 10 million years, a rate that is 6 times faster than the rate of 0.14 chimerical retrogenes per million years in the evolution of the primate lineage toward humans $[29,38]$. If we extended the time to 100 million years, 52 chimerical genes were found and the rate, 0.52 genes per million years, is still about 4 times faster than that found in human. This is among the most rapid rates of generation of chimerical genes, just next to the rice [20].

To know the generation rate of chimerical retrogenes, we plotted the Ks distribution of retrogenes whose Ks was smaller than 1.5. Combined with the synonymous substitution rate of zebrafish genes, our results suggest that the formation of many chimerical retrogenes occurred at a remarkably rapid evolutionary rate, much faster than that of the primate lineage. 


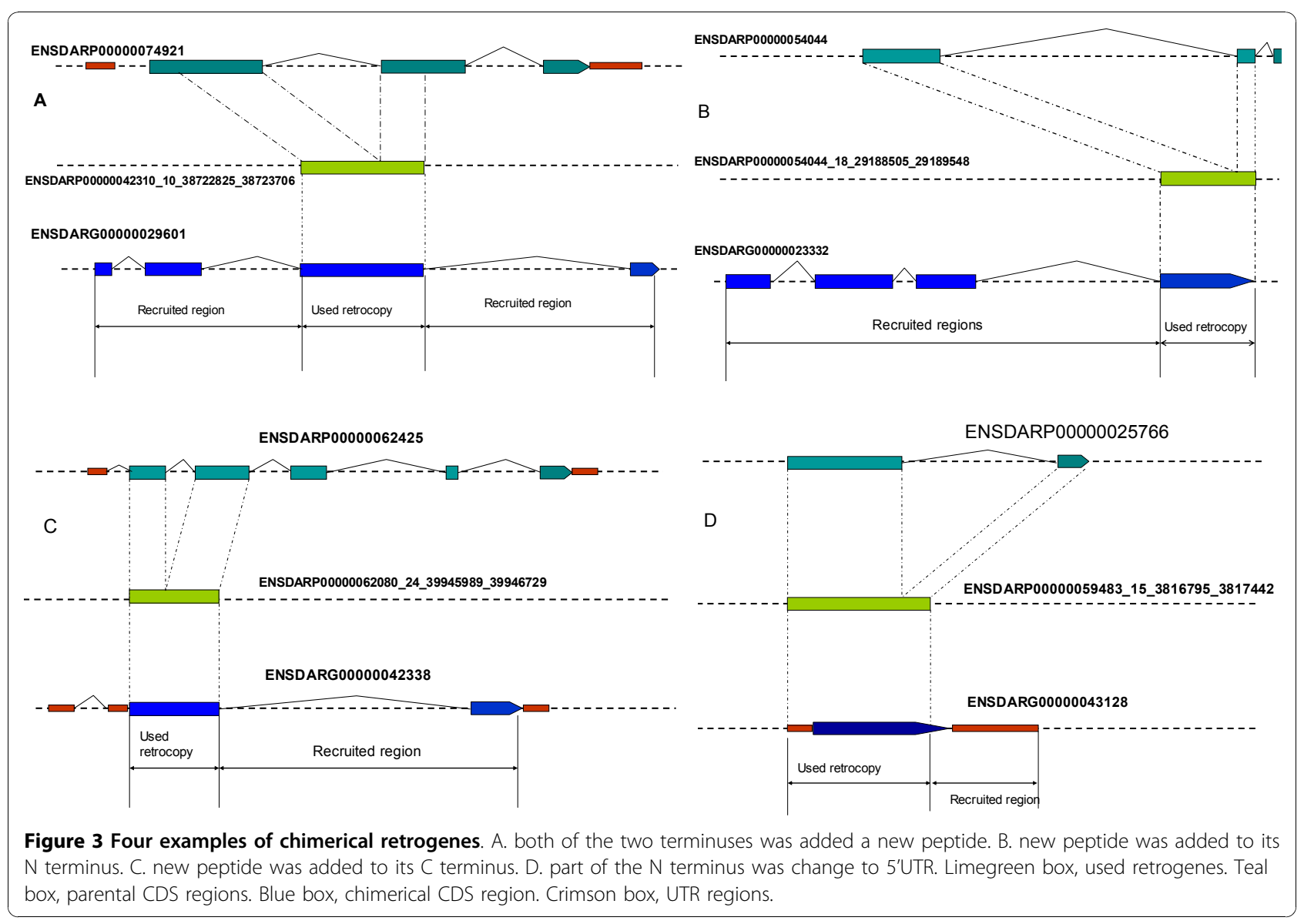

\section{Discussion}

Retrogenes have been seen as evolutionary dead ends with little functional significance for a long time because of their low survival rate [49]. However, when analyzing the zebrafish genome, we found that retroposition was involved in producing a large number of new functional genes. In combining EST with full-length mRNA information, we found some of the new genes might have evolved new functionality during the evolutionary history after the insertion of retrogenes. We used the transcription information and values of $\mathrm{Ka} / \mathrm{Ks}$ as indicators to testify to the functionality of these retrogenes, and found that a large proportion of retrogenes are transcribed, a finding that might indicate their functionality.

In this study, we have found that the transcription of retrogenes is not accidental by using a targeted approach. Our data indicate that retrogene transcription is very common and the transcriptional pattern of zebrafish has been profoundly influenced by natural selection. The finding of a large amount of retrogene transcription is consistent with the fact that a large number of retrogenes are functional [20,50]. Meanwhile, we found the regulatory sources for the transcriptional activity of retrogenes. Some of retrogenes seem to depend on the regulatory sequences of other genes, for example, by recruiting the regulatory sequences of neighboring gene or even directly fusing to host gene. Moreover, some retrogenes used the regulatory sequences of its own sequences. Thus, we predicted that the regions surrounding the position of the inserted retrogenes can influence the expression of the retrogene, and the chimerical retrogene itself can form new gene structure by recruiting new splicing sites.

The ratio of intact retrogenes is very different with that of the human genome, in which intact retrogenes are only a small proportion [38] and which are the result of a burst of young retrogenes in mammal genomes $[29,35]$. One reason may be that although there are many more kinds of LINE-1 in zebrafish than in human, but the total number of LINE-1 is less than that in the human genome $[24,25]$. Another reason might be that the evolutionary rate in teleostei is faster than in mammals [51,52], which makes the pseudogene inserts experience quick turnover (i.e., birth and death of retrotransposons). So, the retropseudogenes with frameshift mutations or premature stop codons are a smaller proportion in zebrafish than in the human genome [29]. 
Chimerical retrogenes have been reported in many species and are considered to be a very important component of protein diversity [53,54]. Recent studies have uncovered several young chimerical retrogene in primates (e.g. TRE2, TRIM5, PMCHL), in Drosophila (e.g. jingwei, sphinx) and in rice, which improves understanding of the molecular process of generating chimerical retrogenes. The systemic search of chimerical retrogenes in our study illustrates that their formation is driven not only by the merging of retrogene and by existing unrelated regions but also from inside the retrogene.

Our results suggest that many new chimerical retrogenes may have originated in zebrafish at a remarkably rapid evolutionary rate 6 times faster than the evolution of the primate lineage toward humans. This tentative estimate represents a lower bound for two reasons. First, we only searched the intact retrocopies for the chimerical genes, although new chimerical genes may have emerged from truncated coding regions [14]. It is also known that new splicing signals in a coding region that contains premature stop codons or frameshifts may evolve to form a new intron or to generate chimerical genes with "host" genes [14]. Second, duplicated "retropseudogenes" may play functional roles by means of their RNA regulating closely related paralogous genes $[55,56]$.

\section{Conclusions}

To examine the birth of these putative retrogenes, we have developed a stringent pipeline for the annotation of retrogenes and retropseudogenes in the zebrafish genome, from which we obtained 652 retrocopies. By using the EST and mRNA as expression evidence, we found that the majority of retrocopies were transcribed. Combined with the evolutionary analysis, we predicted that many retrocopies were functional genes. In addition, 95 retrogenes have recruited new exons or sequences from flanking regions; generating large numbers of chimerical genes, suggesting that gene origination through retroposition is ongoing, with a rate 6 times faster than the rate in humans. This indicates that the functional retrogenes have kept the zebrafish genome in constant flux. In addition retrogenes play an important role in the genome evolution and the retrotransposition provides a strong force for the adaption and speciation of the teleostei fish.

\section{Methods}

\section{Defining the zebrafish retrogenes}

For the zebrafish genome sequence, all annotated peptide sequences and expression data were downloaded from the Ensembl http://www.ensembl.org database (version: Ensembl 50). To define the zebrafish retrogenes, we used a method similar to the one used in identifying the retrogenes in the human genome [29]. To screen for retrogenes, the 31743 annotated protein sequences were used as queries to search against the whole genome sequence in translated similarity using TblastN [30] with an E-value threshold at 10-3. Adjacent homology matches were merged together using Perl scripts, combing only nearby matches (distance < $40 \mathrm{bp}$ ) that were not likely separated by introns. The merged target sequences and the query were thought as true if they, on the amino acid level, had significant similarity (identity $>50 \%$ ) and overlapped with one another more than $70 \%$ of their sequence's length (at least 50 amino acids). GeneWise with default setting and a filtration score at 35 was then used for defining the intron-exon boundary of the merged target sequences. Next, FASTA [32] was used to perform similarity searches of merged target sequences against all Ensembl genes(intron-containing and intronless). We kept only copies in which the closest match was an Ensembl peptide with multiple coding exons (putative parental genes). Merged sequences for which the closest match was an intronless gene were excluded from the data (e.g. intronless gene such as olfactory genes). In this step, we also discarded retrocopies that originated from the duplication of other retrocopies. To confirm the absence of introns in these retrocopies, two GeneWise processes were carried out. First GeneWise was conducted between the protein sequence of putative parental gene and its genomic sequence, which provide all the intron position and length of putative parental gene. Second GeneWise was conducted between the protein sequence of the putative parental gene and its retrocopy (generally, there should not be any intron in this result). By manually checking the two results, we confirm the retrocopy as a real retrocopy if the introns in first result were lost in the second result. This produced 652 retrocopies. Out of the 652 retrocopies, 212 were defined as retropseudogenes with the occurrence of either frameshift mutations or premature stop codons. $\mathrm{Ka}$ and $\mathrm{Ks}$ substitution rates and $\mathrm{Ka} / \mathrm{Ks}$ ratios were calculated using the Ka_Ks calculator program following the LPB methods [57] between the retrogenes and their intron-containing parental genes.

\section{Identification of chimerical retrogenes}

In order to find chimerical retrogenes from all of the retrogenes, we first obtained the genome position of retrogenes and their parental genes as well as all the Ensembl transcripts using the BLAT program[58]. All of the position of the transcripts and retrogenes were obtained, and the retrogenes that overlapped with the transcripts were kept for further analysis. Second, we only kept the transcript that both overlapped with the retrogene and its parental gene for less than $80 \%$ of the 
length of their sequence, and we defined the transcript as a chimeric gene.

\section{Transcription analysis of the retrogenes}

All of the ESTs downloaded from the University of California, Santa Cruz, database (assembly June 2009) were mapped to the zebrafish genome. The map result only kept the best BLAT hit of each EST as well as other hits that had an identity value in the nucleotide that falls less than $0.5 \%$ of the best hit and had at least $96 \%$ nucleotide identity with the genome sequence. To properly discriminate the transcription between the parental gene and retrogene, we proceeded as follows: 1)we only kept the ESTs mapped to a unique location on the genome matched in the University of California, Santa Cruz database criteria; that aligned with the genome sequence of $>100 \mathrm{bp}$; and that had a nucleotide identity of $>97 \%$. 2) Among these ESTs, we only preserved the ESTs that produced an alignment with a genomic sequence that overlapped with a retrogene. In addition, we used ESTs as evidence to support multiexonic transcripts (retrogenes with gene fusions and new exons). We thought of an EST as confirmative evidence if it aligned both with a retrogene-derived and a non-retrogene-derived exon.

\section{Level of transcriptional activity surrounding retrogenes}

Based on the BLAT results of all ESTs to the genome, we excluded ESTs mapped within the 2-kb flanking sequences of retrogenes, and counted the numbers of ESTs aligned with the retrogenes' 40 -kb flanking sequences. We thought that the number obtained was an indicator of the level of transcriptional activity in zebrafish genomic regions surrounding retrogenes.

\section{Distance to closest gene}

We obtained the position of the retrogenes and Ensembl transcripts (start and end) by BLAT and computed the minimal distance between a retrogene and its neighboring gene. We did not consider the orientations of the retrogenes and the Ensembl transcripts for this analysis (transcripts on the sense or anti-sense strands of the retrogenes were treated equally). Ensembl transcripts that overlapped with the annotated retrogenes were removed in this analysis.

\section{Additional material}

Additional file 1: The 652 retrogenes identified in this study Additional file 2: The 437 retrocopies with at least one EST supported.

Additional file 3: The 95 chimerical retrogenes identified in this study.

\section{Acknowledgements}

We are very grateful to Wenjing Tao, Baocheng Guo, Wei Chi for their helpful comments and suggestions that greatly improved the manuscript. This work was supported by the Natural Science Foundation on China (Grant No. 2007CB411601) to Shunping He.

\section{Author details}

${ }^{1}$ The Key Laboratory of Aquatic Biodiversity and Conservation of Chinese Academy of Sciences, Institute of Hydrobiology, Chinese Academy of Sciences, Wuhan 430072, PR China. ${ }^{2}$ Graduate University of the Chinese Academy of Sciences, Beijing 100039, PR China. ${ }^{3}$ Department of Ecology and Evolution, University of Chicago, Chicago 60637, Illinois.

\section{Authors' contributions}

BF developed the algorithm, carried out the molecular genetic studies, performed the sequence alignment and drafted the manuscript. MC participated in algorithm development. MZ participated in the sequence alignment and the data analysis. ML participated in the design of the study. $\mathrm{SH}$ conceived of the study, participated in its design and coordination and helped to draft the manuscript. All authors read and approved the final manuscript.

Received: 30 May 2010 Accepted: 24 November 2010

Published: 24 November 2010

\section{References}

1. Li W-H: Molecular Evolution. Sunderland, MA.: Sinauer Associates; 1997.

2. Brosius J: Retroposons-seeds of evolution. Science (New York, NY) 1991, 251(4995):753

3. Long $M$, Wang $W$, Zhang J: Origin of new genes and source for $\mathrm{N}-$ terminal domain of the chimerical gene, jingwei, in Drosophila. Gene 1999, 238(1):135-141.

4. Long $\mathrm{M}$, Langley $\mathrm{CH}$ : Natural selection and the origin of jingwei, a chimeric processed functional gene in Drosophila. Science (New York, NY) 1993, 260(5104):91-95.

5. Wang W, Brunet FG, Nevo E, Long M: Origin of sphinx, a young chimeric RNA gene in Drosophila melanogaster. Proceedings of the National Academy of Sciences of the United States of America 2002, 99(7):4448-4453.

6. Damert A, Raiz J, Horn AV, Lower J, Wang H, Xing J, Batzer MA, Lower R, Schumann GG: 5'-Transducing SVA retrotransposon groups spread efficiently throughout the human genome. Genome Res 2009, 19(11):1992-2008.

7. Buzdin A, Gogvadze E, Kovalskaya E, Volchkov P, Ustyugova S, Illarionova A, Fushan A, Vinogradova T, Sverdlov E: The human genome contains many types of chimeric retrogenes generated through in vivo RNA recombination. Nucleic acids research 2003, 31(15):4385-4390.

8. Buzdin A, Ustyugova S, Gogvadze E, Vinogradova T, Lebedev Y, Sverdlov E: A new family of chimeric retrotranscripts formed by a full copy of U6 small nuclear RNA fused to the $3^{\prime}$ terminus of I1. Genomics 2002, 80(4):402-406.

9. Bantysh $O B$, Buzdin AA: Novel family of human transposable elements formed due to fusion of the first exon of gene MAST2 with retrotransposon SVA. Biochemistry 2009, 74(12):1393-1399.

10. Hancks DC, Ewing AD, Chen JE, Tokunaga K, Kazazian HH Jr: Exon-trapping mediated by the human retrotransposon SVA. Genome Res 2009, 19(11):1983-1991.

11. Betran E, Thornton K, Long M: Retroposed new genes out of the $\times$ in Drosophila. Genome Res 2002, 12(12):1854-1859.

12. Nisole S, Lynch C, Stoye JP, Yap MW: A Trim5-cyclophilin A fusion protein found in owl monkey kidney cells can restrict HIV-1. Proceedings of the National Academy of Sciences of the United States of America 2004 101(36):13324-13328.

13. Zhang J, Dean AM, Brunet F, Long M: Evolving protein functional diversity in new genes of Drosophila. Proceedings of the National Academy of Sciences of the United States of America 2004, 101(46):16246-16250.

14. Long $M$, Betran $E$, Thornton $K$, Wang $W$ : The origin of new genes: glimpses from the young and old. Nature reviews 2003, 4(11):865-875. 
15. Emerson JJ, Kaessmann H, Betran E, Long M: Extensive gene traffic on the mammalian $\times$ chromosome. Science (New York, NY) 2004, 303(5657):537-540

16. Krasnov AN, Kurshakova MM, Ramensky VE, Mardanov PV, Nabirochkina EN, Georgieva SG: A retrocopy of a gene can functionally displace the source gene in evolution. Nucleic acids research 2005, 33(20):6654-6661.

17. Bai Y, Casola C, Feschotte C, Betran E: Comparative genomics reveals a constant rate of origination and convergent acquisition of functional retrogenes in Drosophila. Genome biology 2007, 8(1):R11.

18. Zhang Y, Wu Y, Liu Y, Han B: Computational identification of 69 retroposons in Arabidopsis. Plant physiology 2005, 138(2):935-948.

19. $\mathrm{Yu} Z$, Morais D, Ivanga M, Harrison PM: Analysis of the role of retrotransposition in gene evolution in vertebrates. BMC bioinformatics 2007, 8:308.

20. Wang W, Zheng H, Fan C, Li J, Shi J, Cai Z, Zhang G, Liu D, Zhang J, Vang $S$, et al: High rate of chimeric gene origination by retroposition in plant genomes. Plant Cell 2006, 18(8):1791-1802.

21. Bellingham J, Tarttelin EE, Foster RG, Wells DJ: Structure and evolution of the teleost extraretinal rod-like opsin (errlo) and ocular rod opsin (rho) genes: is teleost rho a retrogene? Journal of experimental zoology Part $B$ 2003, 297(1):1-10.

22. Esnault C, Maestre J, Heidmann T: Human LINE retrotransposons generate processed pseudogenes. Nature genetics 2000, 24(4):363-367.

23. Furano AV: The biological properties and evolutionary dynamics of mammalian LINE-1 retrotransposons. Progress in nucleic acid research and molecular biology 2000, 64:255-294.

24. Furano AV, Duvernell DD, Boissinot S: L1 (LINE-1) retrotransposon diversity differs dramatically between mammals and fish. Trends Genet 2004, 20(1):9-14

25. Volff JN, Bouneau L, Ozouf-Costaz C, Fischer C: Diversity of retrotransposable elements in compact pufferfish genomes. Trends Genet 2003, 19(12):674-678

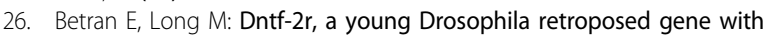
specific male expression under positive Darwinian selection. Genetics 2003, 164(3):977-988

27. Betran $E$, Wang W, Jin L, Long M: Evolution of the phosphoglycerate mutase processed gene in human and chimpanzee revealing the origin of a new primate gene. Molecular biology and evolution 2002, 19(5):654-663.

28. Burki F, Kaessmann H: Birth and adaptive evolution of a hominoid gene that supports high neurotransmitter flux. Nature genetics 2004, 36(10):1061-1063.

29. Marques AC, Dupanloup I, Vinckenbosch N, Reymond A, Kaessmann H: Emergence of young human genes after a burst of retroposition in primates. PLoS biology 2005, 3(11):e357.

30. Altschul SF, Madden TL, Schaffer AA, Zhang J, Zhang Z, Miller W, Lipman DJ: Gapped BLAST and PSI-BLAST: a new generation of protein database search programs. Nucleic acids research 1997, 25(17):3389-3402.

31. Birney E, Clamp M, Durbin R: GeneWise and Genomewise. Genome research 2004, 14(5):988-995.

32. Pearson WR: Rapid and sensitive sequence comparison with FASTP and FASTA. Methods in enzymology 1990, 183:63-98.

33. Yeo $G$, Hoon $S$, Venkatesh B, Burge CB: Variation in sequence and organization of splicing regulatory elements in vertebrate genes. Proceedings of the National Academy of Sciences of the United States of America 2004, 101(44):15700-15705.

34. Kruiswijk CP, Hermsen TT, Westphal AH, Savelkoul HF, Stet RJ: A novel functional class I lineage in zebrafish (Danio rerio), carp (Cyprinus carpio), and large barbus (Barbus intermedius) showing an unusual conservation of the peptide binding domains. J Immunol 2002, 169(4):1936-1947.

35. Pan $D$, Zhang $L$ : Burst of young retrogenes and independent retrogene formation in mammals. PloS one 2009, 4(3):e5040.

36. Reinartz J, Bruyns E, Lin JZ, Burcham T, Brenner S, Bowen B, Kramer M, Woychik R: Massively parallel signature sequencing (MPSS) as a tool for in-depth quantitative gene expression profiling in all organisms. Briefings in functional genomics \& proteomics 2002, 1(1):95-104.

37. Harbers $M$, Carninci P: Tag-based approaches for transcriptome research and genome annotation. Nature methods 2005, 2(7):495-502.

38. Vinckenbosch N, Dupanloup I, Kaessmann H: Evolutionary fate of retroposed gene copies in the human genome. Proceedings of the
National Academy of Sciences of the United States of America 2006 103(9):3220-3225.

39. Harrison PM, Zheng D, Zhang Z, Carriero N, Gerstein M: Transcribed processed pseudogenes in the human genome: an intermediate form of expressed retrosequence lacking protein-coding ability. Nucleic acids research 2005, 33(8):2374-2383.

40. Wang $\mathrm{W}, \mathrm{Yu} \mathrm{H}$, Long M: Duplication-degeneration as a mechanism of gene fission and the origin of new genes in Drosophila species. Nature genetics 2004, 36(5):523-527.

41. Zhu Z, Zhang $Y$, Long M: Extensive structural renovation of retrogenes in the evolution of the Populus genome. Plant physiology 2009, 151(4):1943-1951.

42. Torrents D, Suyama M, Zdobnov E, Bork P: A genome-wide survey of human pseudogenes. Genome Res 2003, 13(12):2559-2567.

43. Nekrutenko A, Makova KD, Li WH: The $K(A) / K(S)$ ratio test for assessing the protein-coding potential of genomic regions: an empirical and simulation study. Genome Res 2002, 12(1):198-202.

44. Moore RC, Purugganan MD: The early stages of duplicate gene evolution Proceedings of the National Academy of Sciences of the United States of America 2003, 100(26):15682-15687.

45. Kazazian HH Jr: Mobile elements: drivers of genome evolution. Science (New York, NY) 2004, 303(5664):1626-1632.

46. Sayah DM, Sokolskaja E, Berthoux L, Luban J: Cyclophilin A retrotransposition into TRIM5 explains owl monkey resistance to HIV-1. Nature 2004, 430(6999):569-573.

47. Brosius J, Gould SJ: On "genomenclature": a comprehensive (and respectful) taxonomy for pseudogenes and other "junk DNA". Proceedings of the National Academy of Sciences of the United States of America 1992, 89(22):10706-10710.

48. Brosius J: Many G-protein-coupled receptors are encoded by retrogenes. Trends Genet 1999, 15(8):304-305.

49. Robinson R: Retrocopied Genes May Enhance Male Fitness. PLoS biology 2005.

50. Baertsch R, Diekhans M, Kent WJ, Haussler D, Brosius J: Retrocopy contributions to the evolution of the human genome. BMC genomics 2008, 9:466.

51. Ravi $V$, Venkatesh B: Rapidly evolving fish genomes and teleost diversity. Current opinion in genetics \& development 2008, 18(6):544-550.

52. Jaillon O, Aury JM, Brunet F, Petit JL, Stange-Thomann N, Mauceli E, Bouneau L, Fischer C, Ozouf-Costaz C, Bernot A, et al: Genome duplication in the teleost fish Tetraodon nigroviridis reveals the early vertebrate proto-karyotype. Nature 2004, 431(7011):946-957.

53. Patthy L: Genome evolution and the evolution of exon-shuffling-a review. Gene 1999, 238(1):103-114.

54. Long $M$, Deutsch $M$, Wang $W$, Betran $E$, Brunet FG, Zhang J: Origin of new genes: evidence from experimental and computational analyses. Genetica 2003, 118(2-3):171-182.

55. Podlaha O, Zhang J: Nonneutral evolution of the transcribed pseudogene Makorin1-p1 in mice. Molecular biology and evolution 2004, 21(12):2202-2209.

56. Hirotsune S, Yoshida N, Chen A, Garrett L, Sugiyama F, Takahashi S, Yagami K, Wynshaw-Boris A, Yoshiki A: An expressed pseudogene regulates the messenger-RNA stability of its homologous coding gene. Nature 2003, 423(6935):91-96.

57. Li WH: Unbiased estimation of the rates of synonymous and nonsynonymous substitution. Journal of molecular evolution 1993, 36(1):96-99.

58. Kent WJ: BLAT-the BLAST-like alignment tool. Genome research 2002 12(4):656-664

doi:10.1186/1471-2164-11-657

Cite this article as: Fu et al:: The rapid generation of chimerical genes expanding protein diversity in zebrafish. BMC Genomics 2010 11:657. 